\title{
EDITORIAL
}

\section{SETTING NEW STANDARDS}

\author{
Agreeing on global standards for depositing clinical trial data could solve the current impasse.
}

In the November 2004 issue of Nature Reviews Drug Discovery, our editorial highlighted the increasing likelihood of clinical trial registration becoming commonplace for pharmaceutical companies. One year on, this is closer to being reality: the Fair Access to Clinical Trials Act is pending in the US; the International Committee of Medical Journal Editors (ICMJE) has published guidelines for the publication of clinical trial data; the World Health Organization (WHO) and others have established guidelines for information that the registries should contain; and several online clinical trial resources have sprung up, including the planned open-access Global Trial Bank and the pharmaceutical industry's own efforts, including that launched recently by the International Federation of Pharmaceutical Industries and Associations (IFPMA).

Developing a global, unbiased and authoritative clinical trial registry with enough functionality to meet the needs of stakeholders, from physicians and scientists to patients, was never expected to be an easy task. In October 2004, the Canadian Institute of Health Research held an open meeting in Ottawa for medical journal editors, investigators, industry representatives, policymakers and consumers to discuss a set of desired principles for a clinical trial registry, now referred to as the Ottawa Statement. Meanwhile, the WHO formally launched its International Clinical Trials Registry Platform (ICTRP) project earlier this year, and in doing so outlined a 'minimal dataset' of 20 trial characteristics that should be included.

However, despite the shared dedication of these parties and the pharmaceutical industry to developing a useful trial registry, key issues are still to be resolved. The WHO minimal dataset falls some way short of the Ottawa Group's recommendations, and there is disagreement between what the Ottawa Group and the WHO deem to be required information, and what the pharmaceutical industry is willing to disclose. Specifically, the IFPMA does not agree that the title of the study, the intervention, the target sample size or the primary and secondary outcomes should be included in trial registration. But critics have pointed out recently that this leaves very little useful information about the nature of the trial ${ }^{1}$. Furthermore, where industry has cooperated with the FDA's
ClinicalTrials.gov database, it has been criticized for not providing meaningful information ${ }^{1}$.

One issue that is confounding progress is how the purpose of the registry relates to the information it holds. Although recent efforts will facilitate patient recruitment, the major drive for a registry arose following calls for companies to disclose all trial data, including negative results. It is not apparent that existing or proposed registries will enable this. And despite the criteria set by the ICMJE for publication of trial data, and a legal requirement for companies to register their trial, it is still not mandatory for a company to disclose all of its relevant trial data.

IFPMA says it is prepared to explore the concept of placing additional proprietary information into a repository that could be accessible for medical editors during peer review of trial data, and will make full details of the trial results publicly available once the drug has been on the market for a year. But questions still remain as to who will police this post-approval publication, and whether this goes far enough to stem criticism of 'publication bias'.

Another issue is the type and amount of trial data and how this will be formatted, bearing in mind the diversity of end-users. As several different databases and online journals are launched, their value will only be realized if guidelines are established for the amount, type and format of the data that is submitted to ensure it is easily searchable.

Clearly, there are still many challenges to improving the transparency of clinical trial data. The most logical first step would be to clarify the minimal dataset that represents a valid registered trial. To this end, an 'open comments' section is currently available on the WHO's website and, as we go to press, the Ottawa Group is continuing the global dialogue by holding its next meeting in Australia. Although the reluctance of the industry to disclose details of trial protocols is understandable, discussion with the WHO seems important in view of the continued stance of medical journals to only recognize WHO-approved trial registration. With appropriate standards, the potential of an invaluable resource for all who are involved in, and benefit from, the development of new drugs might soon be realized.

1. Krleža-Jeric, K. Clinical trial registration: the differing views of the industry, the WHO, and the Ottawa Group. PLoS Med. 2, e378 (2005) 